\title{
Importance of complete blood count parameters and neutrophil-to-lymphocyte ratio in central and peripheral vertigo
}

\author{
(D) Sedat Bilge
}

University of Health Sciences Turkey, Gülhane Faculty of Medicine, Department of Emergency Medicine, Ankara, Turkey

\section{Date submitted:}

05.09.2019

Date accepted:

07.11.2019

Online publication date:

15.03.2020

\section{Corresponding Author:}

Sedat Bilge MD, University of Health

Sciences Turkey, Gülhane Faculty of

Medicine, Department of Emergency

Medicine, Ankara, Turkey

sedatotuz@gmail.com

ORCID:

orcid.org/0000-0002-0669-2859

Keywords: Central vertigo, complete blood count, neutrophil-lymphocyte ratio, peripheral vertigo

\begin{abstract}
Aim: The present study investigated the diagnostic value of complete blood count (CBC) parameters, neutrophil-to-lymphocyte (NLR), mean platelet volume-to-platelet (MPV/PLT), and platelet-to-lymphocyte (PLR) ratios in patients with central vertigo (CV) compared to the value in patients with peripheral vertigo (PV).

Methods: This retrospective study included 31 patients with $C V$ and 132 patients with PV. CBC parameters and NLR, MPV/PLT, and PLR were statistically analyzed in all patients with vertigo.

Results: Statistically significant differences were found between the CV and PV groups for white blood cell (WBC) count $9.55 \pm 2.33$ and $8.24 \pm 2.34 \times 10^{6} / \mathrm{mm}^{3}(p=0.006)$, NLR $3.58 \pm 2.16$ and $2.96 \pm 2.47(p=0.014)$, neutrophil count $6.49 \pm 2.38$ and $5.27 \pm 2.23 \times 10^{6} / \mathrm{mm}^{3}(p=0.007)$, and monocyte count $0.68 \pm 0.24$ and $0.55 \pm 0.21 \times 106 / \mathrm{mm}^{3}(p=0.003)$.
\end{abstract}

Conclusion: WBC, neutrophil, and monocyte counts and NLR may be useful in the differential diagnosis of CV and PV.

\section{Introduction}

Vertigo is a common cause of admission to health centers, from family health centers to emergency departments (EDs) (1). It is classified into two main groups according to its underlying factors: central vertigo (CV) and peripheral vertigo (PV).

Differentiation between $\mathrm{CV}$ and $\mathrm{PV}$ requires clinical and laboratory examinations of acute cardiac, metabolic, toxic, local, and systemic infection and exclusion of ischemic and hemorrhagic disorders related to the central nervous system. The importance of physical examinations alone in the diagnosis of patients with acute vestibular syndrome is well known in EDs and is tested using head impulse, nystagmus, and test of skew (HINTS), which is a three-step examination (2). HINTS was shown to be more useful than magnetic resonance diffusionweighted imaging (DWI) for differentiating between $\mathrm{CV}$ and PV. However, ED physicians may prefer to employ a brain computed tomography (CT) scan and/or DWI if there is suspicion of a central event related to ischemia or bleeding in the posterior fossa structures in vertigo patients (3).

Acute ischemic stroke (AIS) is a type of brain injury that develops as a result of thrombotic or embolic occlusion of the brain's blood flow and is affected by inflammatory processes (4). Complete blood count (CBC) parameters and the proportional values obtained from these parameters were reported to have diagnostic values as biomarkers (5-8). From the perspective of an ED physician, tests such as a CBC are simply components of routine practice and often have no direct impact on ED applications or diagnostic/prognostic value (9). Furthermore, the combined assessment of all laboratory results and clinical findings lead to preference for expensive, often unnecessary, central imaging methods.

The present study investigated the role of $\mathrm{CBC}$ parameters and neutrophil-to-lymphocyte ratio (NLR), platelet-to-lymphocyte ratio (PLR), and mean platelet volume-to-MPV/PLT) ratio, which 
are calculated from CBC parameters, in the differential diagnosis between $\mathrm{CV}$ and $\mathrm{PV}$.

\section{Methods}

This retrospective study was designed in the ED of University of Health Sciences Turkey, Gülhane Training and Research Hospital, which is a tertiary hospital of the University of Health Sciences Turkey. The study was approved by the Ethics Committee for the Non-invasive Research at the Gülhane University of Health Sciences Turkey (no: 18/366). Informed consent was not received due to the retrospective nature of the study.

\section{Study design and patient selection}

Patients $>18$ years old who had been to the ED in Gülhane Training and Research Hospital between August 2016 and December 2018 with a pre-diagnosis of vertigo and who had undergone $\mathrm{CBC}$ tests and magnetic resonance imaging (MRI) examinations were screened retrospectively using the hospital information management system (FONET, Information Technology Incorporation, Turkey). Age, sex, DWI reports requested by the ED, all consultation results, and hospitalization and discharge information of the screened patients were examined using the patients' files.

Patients with CV and PV were identified, and CBC subparameters including NLR, MPV/PLT ratio, and PLR were statistically analyzed in all the patients. Patients were divided into two groups, as those with $\mathrm{CV}$ and those with $\mathrm{PV}$, after definitive diagnoses using total examinations.

Exclusion criteria were as follows: patients $<18$ years old, patients with supratentorial ischemic stroke, pregnancy, history of recently performed surgery, trauma, major systemic diseases (renal or hepatic disease, hematologic and autoimmune disorder) that may affect NLR and PLR levels, evidence of infection (excluding ear, nose, and throat and central nervous system-related infections), and use of drugs (iron preparations, chemotherapeutic agents, vitamins, and corticosteroids) that could influence CBC measurements.

\section{Power analysis}

A minimum of 27 patients was calculated to be required for each group based on the findings from a previous study [NLR value for AIS of $4.87 \pm 3.48$ (7), and NLR value for PV of 2.20 (10) with $80 \%$ power and a two-sided error margin of 0.05 ].

\section{Laboratory analysis}

Blood samples taken for CBC examination in the ED of the hospital were analyzed using Sysmex XN-1000 (Sysmex America Inc., Lincolnshire, IL, USA) and Beckman Coulter UniCel DxH800 (Beckman Coulter, Miami, FL, USA) analyzers. Neutrophils, lymphocytes, PLT, red blood cells, and white blood cells (WBCs) were measured as part of the automated CBC. NLR and PLR were calculated as NLR and PLR, respectively.

\section{Statistical Analysis}

Statistical analyses were performed using SPSS for Windows v18.0 (SPSS Inc., Chicago, IL, USA) software package. Descriptive data were expressed in mean \pm standard deviation. Kolmogorov-Smirnov test was used to evaluate normally distributed data. Student's t-test was used for the comparison of normally distributed paired groups whereas Mann-Whitney $U$ test was employed for non-normally distributed groups. All $p$ values $<0.05$ were considered statistically significant. Receiver operating characteristic (ROC) analysis was performed to identify cutoff thresholds for WBC count, neutrophil, monocyte, and NLR. Sensitivity, specificity, and area under the ROC curve were used for overall estimation of the accuracy in distinguishing CV cases.

\section{Results}

A total of 163 patients underwent MRI examination in addition to $\mathrm{CBC}$ tests. In the $\mathrm{CV}$ group $(n=31)$, only ischemia was detected in the infratentorial region, and all CV patients were hospitalized. Ischemia, hemorrhage, space-occupying lesion, and infection were not detected in any region of the central nervous system in the PV group $(n=132)$, and all PV patients were discharged from the ED.

There was no statistically significant difference for age between the CV and PV groups (62 \pm 16 and 59 \pm 17 , respectively; $p=0.35)$. Sex distribution analysis showed $52 \%$ of the patients in the CV group were male $(n=16)$ and $48 \%$ were female $(n=15)$. In the PV group, $46 \%$ of the patients were male $(n=61)$ and $54 \%$ were female $(n=71)$. There was no statistically significant difference between the groups regarding sex $(p=0.37)$.

WBC, neutrophil, and monocyte counts were significantly higher in the CV group compared to those in the PV group $(p=0.006, p=0.007$, and $p=0.003$, respectively). There was no significant difference between the two groups for lymphocyte and PLT counts and MPV $(p=0.67, p=0.21$, and $p=0.72$, respectively $)$ (Table 1).

NLR was significantly higher in the CV group compared to that in the PV group $(p=0.014)$. However, there was no statistically significant difference between the groups in terms of PLR and MPV/PLT ratio ( $p=0.273$ and $p=0.453$, respectively) (Table 2).

A cutoff value of 8.55 for WBC count, which was derived from the ROC analysis, showed sensitivity and specificity values of $74.19 \%$ and $62.88 \%$, respectively, for the prediction of CV. However, the area under the curve (AUC) value for NLR to distinguish between $\mathrm{CV}$ and PV groups was 0.642. NLR had the best cutoff value of 2.25 , with a sensitivity of $61.29 \%$ and a specificity of $56.06 \%$. On the other hand, the AUC value for 
monocytes to distinguish between $\mathrm{CV}$ and $\mathrm{PV}$ patients was 0.676 , with a cutoff value of 0.56 , sensitivity of $74.19 \%$, and specificity of $54.55 \%$. Other CBC parameters and ROC analysis related to NLR are shown in Table 3. ROC analysis is shown separately in Figure 1.

\section{Discussion}

Vertigo is a common symptom that may be encountered in many diseases, including most benign disorders. However, to avoid misdiagnosis, effective differential diagnosis of vertigo is required, particularly in patients admitted to the ED. Vanni et al. (11) performed CV-PV differential diagnosis using clinical examination only and showed that $16.4 \%$ of patients were diagnosed with $\mathrm{CV}$ whereas $33.4 \%$ were diagnosed with PV. The authors also emphasized that CT and MRI examination rates were low among $\mathrm{CV}$ patients, and patients were hospitalized on the basis of physical examination alone. Consequently, the use of adjuvant diagnostic tools with clinical examination was suggested to provide an optimal benefit for the differential diagnosis between $\mathrm{CV}$ and PV. The results of the present study indicated that WBC, neutrophil, and monocyte counts and NLR might contribute to the differential diagnosis of CV and PV and hospitalization (independent of clinical findings).

It was previously reported that increased WBC count might be an indicator of high risk for acute myocardial infarct and acute cerebrovascular events $(12,13)$. Zheng et al. (14) reported that increased WBC count on admission was related

Table 1. Comparison of complete blood count parameters between the central and peripheral vertigo groups

\begin{tabular}{|llll|}
\hline & $\begin{array}{l}\text { CV }(\mathbf{n = 3 1} \\
\text { Mean } \pm \text { SD }\end{array}$ & $\begin{array}{l}\text { PV }(\mathbf{n = 1 3 2}) \\
\text { Mean } \pm \text { SD }\end{array}$ & p value* \\
\hline WBCs $\left(\times 10^{6} / \mathrm{mm}^{3}\right)$ & $9.55 \pm 2.33$ & $8.24 \pm 2.34$ & 0.006 \\
\hline Neutrophils $\left(\times 10^{6} / \mathrm{mm}^{3}\right)$ & $6.49 \pm 2.38$ & $5.27 \pm 2.23$ & 0.007 \\
\hline Lymphocytes $\left(\times 10^{6} / \mathrm{mm}^{3}\right)$ & $2.15 \pm 0.78$ & $2.22 \pm 0.85$ & 0.67 \\
\hline Monocytes $\left(\times 10^{6} / \mathrm{mm}^{3}\right)$ & $0.68 \pm 0.24$ & $0.55 \pm 0.21$ & 0.003 \\
\hline Platelets $(\mathrm{K} / \mathrm{\mu L})$ & $270.23 \pm 67.01$ & $253.46 \pm 66.62$ & 0.21 \\
\hline MPV (fL) & $9.38 \pm 1.66$ & $9.29 \pm 1.17$ & 0.72 \\
\hline $\begin{array}{l}\text { *Student's t-test. } \\
\text { CV: Central vertigo, MPV: Mean platelet volume, PV: Peripheral vertigo, SD: Standard deviation, WBCs: White blood cells }\end{array}$ & \\
\hline
\end{tabular}

Table 2. Comparison of neutrophil-to-lymphocyte ratio, platelet-to-lymphocyte ratio, and mean platelet volume-to-platelet ratio between the central and peripheral vertigo groups

\begin{tabular}{|llll|}
\hline & $\begin{array}{l}\text { CV }(\mathbf{n = 3 1}) \\
\text { Mean } \pm \text { SD }\end{array}$ & $\begin{array}{l}\text { PV }(\mathbf{n = 1 3 2}) \\
\text { Mean } \pm \text { SD }\end{array}$ & p value* \\
\hline NLR & $3.58 \pm 2.16$ & $2.96 \pm 2.47$ & 0.014 \\
\hline PLR & $144.95 \pm 75.49$ & $133.23 \pm 68.28$ & 0.273 \\
\hline MPV/PLT ratio & $0.04 \pm 0.01$ & $0.04 \pm 0.01$ & 0.453 \\
\hline $\begin{array}{l}\text { *Mann-Whitney U test. } \\
\text { CV: Central vertigo, MPV/PLT: Mean platelet volume-to-platelet, NLR: Neutrophil-to-lymphocyte ratio, PLR: Platelet-to-lymphocyte ratio, PV: Peripheral vertigo group, } \\
\text { SD: Standard deviation }\end{array}$ & & \\
\hline
\end{tabular}

Table 3. Receiver operating characteristic curve analysis of complete blood count parameters and neutrophil-tolymphocyte ratio for differentiating between central vertigo and peripheral vertigo

\begin{tabular}{|c|c|c|c|c|c|c|c|}
\hline & Cutoff & AUC & $\begin{array}{l}\text { Sensitivity (\%) } \\
(95 \% \mathrm{Cl})\end{array}$ & $\begin{array}{l}\text { Specificity (\%) } \\
(95 \% \mathrm{Cl})\end{array}$ & $\begin{array}{l}\text { LR+ } \\
(95 \% \mathrm{Cl})\end{array}$ & $\begin{array}{l}\text { LR- } \\
(95 \% \mathrm{Cl})\end{array}$ & p value* \\
\hline $\begin{array}{l}\text { WBCs } \\
\left(\times 10^{6} / \mathrm{mm}^{3}\right)\end{array}$ & 8.55 & 0.675 & $\begin{array}{l}74.19 \\
(55.39-88.14)\end{array}$ & $\begin{array}{l}62.88 \\
(54.04-71.12)\end{array}$ & $\begin{array}{l}2.00 \\
(1.47-2.71)\end{array}$ & $\begin{array}{l}0.41 \\
(0.22-0.76)\end{array}$ & 0.002 \\
\hline $\begin{array}{l}\text { Neutrophils } \\
\left(\times 106 / \mathrm{mm}^{3}\right)\end{array}$ & 5.40 & 0.664 & $\begin{array}{l}74.19 \\
(55.39-88.14)\end{array}$ & $\begin{array}{l}62.12 \\
(53.27-70.42)\end{array}$ & $\begin{array}{l}1.96 \\
(1.45-2.65)\end{array}$ & $\begin{array}{l}0.42 \\
(0.23-0.77)\end{array}$ & 0.005 \\
\hline $\begin{array}{l}\text { Monocytes } \\
\left(\times 106 / \mathrm{mm}^{3}\right)\end{array}$ & 0.56 & 0.676 & $\begin{array}{l}74.19 \\
(55.39-88.14)\end{array}$ & $\begin{array}{l}54.55 \\
(45.65-63.23)\end{array}$ & $\begin{array}{l}1.63 \\
(1.23-2.16)\end{array}$ & $\begin{array}{l}0.47 \\
(0.26-0.88)\end{array}$ & 0.002 \\
\hline NLR & 2.25 & 0.642 & $\begin{array}{l}61.29 \\
(42.19-78.15)\end{array}$ & $\begin{array}{l}56.06 \\
(47.16-64.68)\end{array}$ & $\begin{array}{l}1.39 \\
(0.99-1.96)\end{array}$ & $\begin{array}{l}0.69 \\
(0.43-1.10)\end{array}$ & 0.014 \\
\hline
\end{tabular}

${ }^{*}$ Chi-square test.

AUC: Area under curve, Cl: Confidence interval, LR-: Negative likelihood ratio, LR+: Positive likelihood ratio, NLR: Neutrophil-to-lymphocyte ratio, WBCs: White blood cells 
to poor prognosis at 3 months in patients with ischemic stroke independent of other inflammation factors. Similar to these findings, the present study found the WBC count was higher in CV patients with ischemic stroke compared to that in the PV group.

Ozbay et al. (10) conducted a prospective controlled study and compared WBC, PLT, neutrophil, and lymphocyte counts and MPV and NLR values in PV patients and in the control group. No differences were found between the study groups, and all parameters were within the reference intervals. Furthermore, the sub-parameters of the hemogram (CBC) analysis were all within the normal limits. This was an important and distinctive factor in the exclusion of the effects of additional pathologies due to possible inflammation in our patient groups. Thus, the levels of cell count within the normal limits avoided a possible bias while demonstrating the importance of WBC levels in CV and PV distinction.

Neutrophil levels are expected to increase during inflammatory responses activated by ischemia and reperfusion $(15,16)$. Zhou et al. (17) studied AIS patients undergoing clopidogrel treatment for 90 days and demonstrated that new ischemic strokes developed in patients with high neutrophil levels. In the present study, neutrophil counts were higher in CV patients compared to those in PV patients, which may be because all patients were diagnosed with AIS in the infratentorial region. In addition, the degree of neutrophil accumulation, particularly in the early stages and in regions of cerebral ischemia, correlates with stroke severity and poorer stroke outcomes (18). Therefore, targeting neutrophils to reduce brain injury in ischemic stroke may be beneficial by blocking

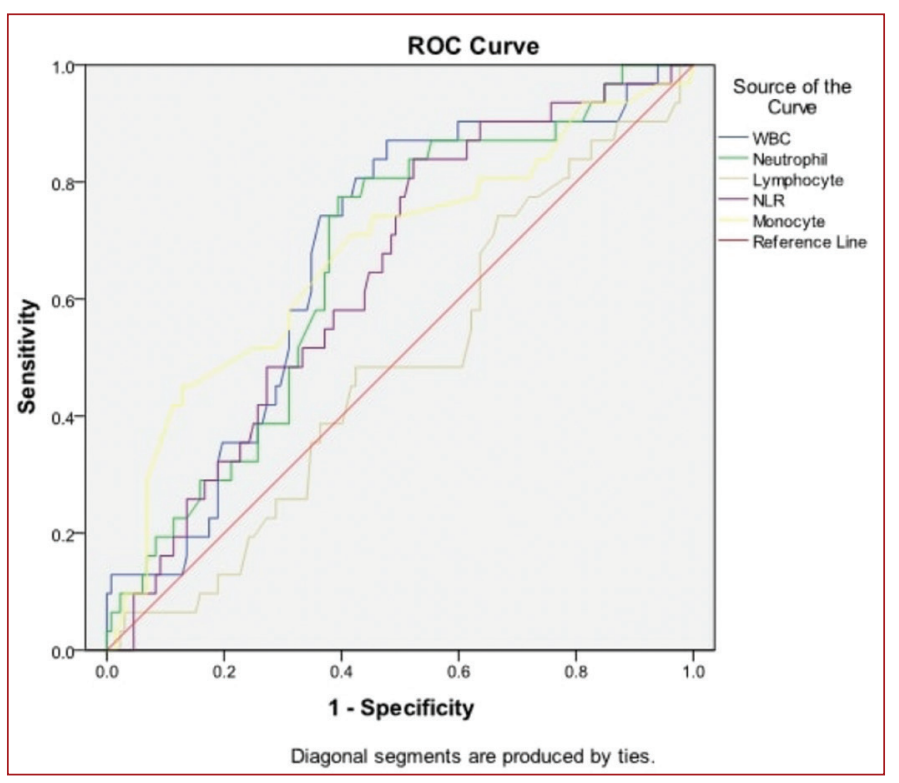

Figure 1. Receiver operating characteristic curves of white blood cells, neutrophils, lymphocytes, neutrophil-to-lymphocyte ratio, and monocytes levels for differentiating between central vertigo and peripheral vertigo

ROC: Receiver operating characteristic, WBC: White blood cells, NLR: Neutrophil-to-lymphocyte ratio neutrophil adhesion to endothelial cells, neutrophil transmigration, and neurovascular interactions (19). The increase in neutrophils observed in patients in the CV group compared to those in the PV group, particularly in the early stages, although within normal limits, justifies the need to investigate the role of antineutrophil therapy in these patient groups.

Following AIS, monocyte levels increase and migrate to the infarct area, contributing to the enlargement of the ischemic lesion $(20,21)$. Bolayır (20) reported that the monocyte count was significantly higher in AIS patients compared to controls. The same study also reported that monocyte count was statistically higher in AIS patients who died within 30 days compared to those who survived. In accordance with these findings, the present study also showed that monocyte levels were significantly higher in $\mathrm{CV}$ patients compared to those in PV patients (Table 1). These similar findings may depend on the early involvement of monocytes in the inflammatory processes of acute brain ischemia.

In the present study, lymphocyte levels were lower but not statistically significant in the CV group compared to those in the PV group. This was attributed to the adaptive lymphocytic response in the late phase of the inflammatory process. Bolayır (20) took blood samples from patients hospitalized in the neurology clinic within $24 \mathrm{~h}$ of hospitalization whereas the present study used blood samples taken at admission to the ED. In this context, the CBC parameters obtained in this study may reflect the early stage of the inflammatory response in AIS patients and are more valuable to initial diagnoses. Some studies have reported decreased levels of lymphocytes in AIS patients after ischemia $(22,23)$. Pagram et al. (23) suggested that lymphocyte levels decreased after the first $24 \mathrm{~h}$ compared to lymphocyte levels at admission in patients with ischemic stroke. In the present study, lymphocyte levels were within the normal limits in both groups, and it was concluded that lymphocyte levels had limited value in differentiating between $\mathrm{CV}$ and $\mathrm{PV}$ patients.

The diagnostic and prognostic values of increased NLR in AIS have previously been demonstrated (16,24-26). In accordance with previous studies, the present study showed increased levels of NLR accompanied by acute ischemia in the infratentorial region in $\mathrm{CV}$ patients. This increase was mainly due to elevated neutrophil counts. The difference in neutrophil levels was more significant compared to that for NLR values. Furthermore, since neutrophil counts were within the normal limits in both groups, they were not considered to have diagnostic value alone. On the other hand, neutrophil counts were significantly higher but within normal limits in CV patients, highlighting the need for a marker that reveals this significant difference in the early stages of the disease. Since there was also no difference between the groups in terms of lymphocyte and PLT counts, PLR was shown to have no 
diagnostic value, and the importance of NLR derived from neutrophil indices became more prominent. Therefore, NLR is recommended as an effective, inexpensive, and fast indicator for the differential diagnosis between $\mathrm{CV}$ and $\mathrm{PV}$ in the early stage of vertigo. In addition, Ozbay et al. (10) were the first to report the relationship between the CBC parameters in PV patients and they showed that NLR in PV patients was higher compared to that in healthy controls. In the present study, NLR was higher in the $\mathrm{CV}$ group than that in the PV group. Although this finding seems to conflict with those of Ozbay et al. (10), the present study showed meaningful results that included CV patients. Moreover, we found significant NLR levels in the CV group compared to those in the PV group.

In a retrospective study by Lok et al. (27), who investigated AIS patients, MPV was found to have no predictive or prognostic value. This study also showed similar findings in CV patients, and it was concluded that MPV had no value regarding the differential diagnosis between CV and PV. Ozbay et al. (10) found that PV patients had PLT counts and MPV within the normal limits, and there was no difference compared to the control group. Consistent with these findings, PLT parameters, including PLT count and MPV, had no value in the diagnosis and differential diagnoses of $C V$ and $P V$ in the present study.

The main limitation of this study is a lack of data for proinflammatory cytokines and/or inflammation markers (e.g., C-reactive protein), which may play a key role in the inflammation process in PV and CV. Another limitation is the relatively small sample size. The present data should, therefore, be interpreted with caution and need to be confirmed in a larger cohort.

\section{Conclusion}

In conclusion, WBC, neutrophil, and monocyte counts and NLR may be helpful in the differential diagnosis between CV and $P V$ in patients who present with the complaints of vertigo to the ED. Regarding the differential diagnosis between $C V$ and $\mathrm{PV}$, the use of simple, easily available, and inexpensive CBC parameters, NLR, and monocyte counts, along with the clinical diagnostic tools, may prevent the unnecessary implementation of advanced and expensive imaging methods such as CT and MRI.

\section{Ethics}

Ethics Committee Approval: The study was approved by the Ethics Committee for the Non-invasive Research at the Gülhane University of Health Sciences Turkey (no: 18/366).

Informed Consent: Informed consent was not received due to the retrospective nature of the study.

Peer-review: Externally and internally peer-reviewed.

Financial Disclosure: The financial support for this study was provided by the investigator.

\section{References}

1. Kerber KA, Meurer WJ, West BT, Fendrick AM. Dizziness presentations in U.S. emergency departments, 1995-2004. Acad Emerg Med. 2008;15:744-750.

2. Newman-Toker DE. Comment: Diagnosing stroke in acute dizziness-Do the "eyes" still have it? Neurology. 2015;85:1877.

3. Perloff MD, Patel NS, Kase CS, Oza AU, Voetsch B, Romero JR. Cerebellar stroke presenting with isolated dizziness: Brain MRI in 136 patients. Am J Emerg Med. 2017;35:1724-1729.

4. Harpaz D, Eltzov E, Seet RCS, Marks RS, Tok AIY. Point-ofCare-Testing in Acute Stroke Management: An Unmet Need Ripe for Technological Harvest. Biosensors (Basel). 2017:7.

5. Fang YN, Tong MS, Sung PH, et al. Higher neutrophil counts and neutrophil-to-lymphocyte ratio predict prognostic outcomes in patients after non-atrial fibrillationcaused ischemic stroke. Biomed J. 2017;40:154-162.

6. Farah R, Samra N. Mean platelets volume and neutrophil to lymphocyte ratio as predictors of stroke. J Clin Lab Anal. 2018:32.

7. Gökhan S, Ozhasenekler A, Mansur Durgun H, Akil E, Ustündag M, Orak M. Neutrophil lymphocyte ratios in stroke subtypes and transient ischemic attack. Eur Rev Med Pharmacol Sci. 2013;17:653-657.

8. Gumus F, Solak I, Eryilmaz MA. The effects of smoking on neutrophil/lymphocyte, platelet/lymphocyte ratios. Bratisl Lek Listy. 2018;119:116-119.

9. Ganti L, Gilmore RM, Weaver AL, Brown RD. Prognostic Value of Complete Blood Count and Electrolyte Panel during Emergency Department Evaluation for Acute Ischemic Stroke. ISRN Stroke. 2013;2013:1-5.

10. Ozbay I, Kahraman C, Balikci HH, Kucur C, Kahraman NK, Ozkaya DP, Oghan F. Neutrophil-to-lymphocyte ratio in patients with peripheral vertigo: a prospective controlled clinical study. Am J Otolaryngol. 2014;35:699-702.

11. Vanni S, Pecci R, Casati C, et al. STANDING, a four-step bedside algorithm for differential diagnosis of acute vertigo in the Emergency Department. Acta Otorhinolaryngol Ital. 2014;34:419-426.

12. Byrne CE, Fitzgerald A, Cannon CP, Fitzgerald DJ, Shields DC. Elevated white cell count in acute coronary syndromes: relationship to variants in inflammatory and thrombotic genes. BMC Med Genet. 2004;5:13.

13. Koren-Morag N, Tanne D, Goldbourt U. White blood cell count and the incidence of ischemic stroke in coronary heart disease patients. Am J Med. 2005;118:1004-1009.

14. Zheng $\mathrm{X}$, Zeng $\mathrm{N}$, Wang $\mathrm{A}$, et al. Prognostic Value of White Blood Cell in Acute Ischemic Stroke Patients. Curr Neurovasc Res. 2018;15:151-157.

15. Bhat T, Teli S, Rijal J, et al. Neutrophil to lymphocyte ratio and cardiovascular diseases: a review. Expert Rev Cardiovasc Ther. 2013;11:55-59. 
16. Lattanzi S, Cagnetti C, Provinciali L, Silvestrini M. Neutrophil-to-Lymphocyte Ratio Predicts the Outcome of Acute Intracerebral Hemorrhage. Stroke. 2016;47:16541657.

17. Zhou S, Cai B, Zhang Y, Wang L, Liu X, Xu G. The Relationship between Neutrophil-to-Lymphocyte Ratio and Aortic Arch Calcification in Ischemic Stroke Patients. J Stroke Cerebrovasc Dis. 2017;26:1228-1232.

18. Akopov SE, Simonian NA, Grigorian GS. Dynamics of polymorphonuclear leukocyte accumulation in acute cerebral infarction and their correlation with brain tissue damage. Stroke. 1996;27:1739-1743.

19. Dimasi D, Sun WY, Bonder CS. Neutrophil interactions with the vascular endothelium. Int Immunopharmacol. 2013;17:1167-1175.

20. Bolayır A. The relationship between lymphocyte/monocyte ratio and short-term mortality in acute ischemic stroke patients. Cumhuriyet Med J. 2018;40:128-134.

21. Liberale L, Montecucco F, Bonaventura A, et al. Monocyte count at onset predicts poststroke outcomes during a 90day follow-up. Eur J Clin Invest. 2017;47:702-710.
22. Kim J, Song TJ, Park JH, et al. Different prognostic value of white blood cell subtypes in patients with acute cerebral infarction. Atherosclerosis. 2012;222:464-467.

23. Pagram H, Bivard A, Lincz LF, Levi C. Peripheral Immune Cell Counts and Advanced Imaging as Biomarkers of Stroke Outcome. Cerebrovasc Dis Extra. 2016;6:120-128.

24. Qun S, Tang Y, Sun J, et al. Neutrophil-To-Lymphocyte Ratio Predicts 3-Month Outcome of Acute Ischemic Stroke. Neurotox Res. 2017;31:444-452.

25. Tokgoz S, Keskin S, Kayrak M, Seyithanoglu A, Ogmegul A. Is neutrophil/lymphocyte ratio predict to short-term mortality in acute cerebral infarct independently from infarct volume? J Stroke Cerebrovasc Dis. 2014;23:2163-2168.

26. Xue J, Huang W, Chen X, Li Q, Cai Z, Yu T, Shao B. Neutrophil-to-Lymphocyte Ratio Is a Prognostic Marker in Acute Ischemic Stroke. J Stroke Cerebrovasc Dis. 2017;26:650-657.

27. Lok U, Gulacti U, Ekmekci B, Bulut T, Celik M. Predictive and prognostic role of mean platelet volume in patients with first-ever acute ischemic stroke. Neurosciences (Riyadh). 2017;22:119-126. 